\title{
Experimentation of an Adaptive and Autonomous RF Signalling Strategy for Detection
}

\author{
Aaron M Jones \\ Radar Sensing Team \\ Defence Science and Technology Laboratory \\ Salisbury, UK \\ Email: ajones9@dstl.gov.uk
}

\author{
Colin P Horne, Hugh D Griffiths \\ Department of Electronic and Electrical Engineering \\ University College London \\ London, UK
}

\author{
Graeme Smith, Adam Mitchell, Peter John-Baptiste \\ Department of Electronic and Electrical Engineering \\ The Ohio State University \\ Columbus, $\mathrm{OH}$
}

\begin{abstract}
In this paper we discuss a straightforward signalling strategy for target detection and shared spectrum usage. We further combine additional practical concerns such as waveform modulus and extended target matched illumination to improve detection performance in dynamic interference environments. The impetus for the straightforward, yet suboptimal, strategy are the low cost drivers for small sensing platforms, such as small unmanned aerial systems (SUAS). Herein, we discuss a complete strategy and algorithm for sensing the environment, interrogating the target response and adapting the transmitted waveform to avoid the primary users while putting more energy on target than previous non-adaptive strategies. We give simulation results and initial experimental results to corroborate the theoretical and simulated findings.
\end{abstract}

\section{INTRODUCTION}

Sensing platforms must contend with an ever increasingly contested/congested electromagnetic spectrum and bandwidth management issues, both of which are finite resources. Additionally, size, weight and power (SWAP) restrictions plague small UAS in terms of equipping them with sensors for particular mission sets or even potential weaponization. However, future SUAS will enable new operational concepts like teaming, swarming and loyal wingman, which allow for a single operator to control large numbers of platforms making these platforms desirable in dynamic environments. Overcoming the competing requirements of high capability and SWAP concerns is an ongoing effort but here will address these concerns with radar transmit signal design for fast adaptation in a highly simplified manner. This will be achieved by increasing the signal-to-interference-and-noise-ratio (SINR), over a fixed waveform, through target matched illumination (TMI) and radio frequency interference (RFI) avoidance while maintaining signal constant modulus (i.e. power efficient) on transmit. Other approaches have tried previously to accomplish signal design under these restrictions, and admittedly can provide better ultimate SINR performance and spectrum shaping [1], but fall short in that the time to converge on a solution and the complexity of the algorithms require increased processing time/capability. Often not practical in computational/power limited situations (such as SUAS) in highly dynamic environments where real-time or near real-time adaptation is required.

It is proposed to use the Error Reduction Algorithm (ERA) [2], [3], for all waveform design and optimization (including a novel method to provide TMI) in this paper. The ERA is a straightforward iterative design method that employs an alternating projections technique to enforce the waveform constraints in the time and Fourier domains (effectively providing modulus and spectrum shaping); respectively as the waveform design improves. The simplicity of the algorithm, first used as a phase retrieval technique for image estimation, makes it suitable for limited processing power. While this is a suboptimal SINR waveform design technique, it could yield faster convergence and simpler implementation over current signal designs techniques.

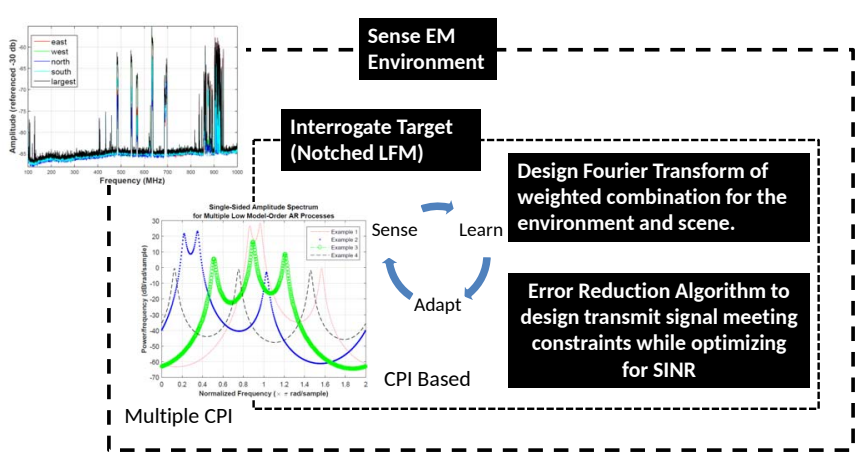

Fig. 1: Proposed adaptive/autonomous signalling strategy hierarchy for interference avoidance and target matched illumination.

\section{A. Technique Description}

First we describe the signal processing technique and waveform design strategy to achieve adaptive implied SINR optimization and interference avoidance. In order to maintain 
control over the simulations and experiment, we generate a synthetic power spectrum to emulate a congested EM environment (we consider these signals to be primary users (PU) and prohibited bands for transmission in the waveform design). Furthermore, to impart a dynamic interference scenario, the model simulates the transition between the radar bearing from one direction to another, where each direction captures a unique interference environment. For example, one could think about the radar changing aspects relative to two major urban areas and the spectrum changing as function of aspect angle. In our adaptive strategy, the spectrum is estimated periodically via a listen activity at the radar.

Next, after interference estimation, a notched linear frequency modulated (LFM) waveform, with notch locations corresponding to PU's bands, is designed and transmitted to interrogate the target. In our scenario we assume an extended target response that can be estimated from the returned signal. Additionally, to further increase the practicality of the scenario, we assert the target response is also dynamic. For example, the target could be changing aspect relative to the radar and thus different features of the target are illuminated. We use a high order auto-regressive model to emulate the target spectral response.

Finally, we combine the estimated interference and target response to generate a Fourier transform mask (FTM) to be used in the adaptive waveform design. To avoid interference, the waveform spectrum is the inverse of a weighted linear combination of the sensed primary users and the extended target response. The ERA is used to design the waveform with constant modulus that is matched to the target for SINR optimization while avoiding RFI. A high level depiction of the strategy can be found in Figure 1.

\section{B. Notation}

We denote column vectors as underlined lowercase letters. The $n^{t h}$ entry of a column vector $\underline{\mathrm{s}}$ is characterized $\underline{\mathrm{s}}_{n}$. Matrices are shown using boldface capital letters. Scalar quantities are as capital letters. The Hermitian operator is given as $(\cdot)^{H}$, while the expectation operator is $\mathbf{E}\{\cdot\}$. Finally, the Hadamard operation is given by the $\odot$ symbol, the convolution operation is $*$, while $\sim$ denotes a probability density.

\section{Preliminaries And Problem Formulation}

We have a transmit waveform model of

$$
\underline{\mathrm{s}}=\underline{\alpha} \odot \exp \{j \underline{\phi}\}
$$

where $\underline{s} \in \mathbb{C}^{M \times 1}, \underline{\alpha}$ is the waveform amplitude and $\phi$ the phase component. In the presence of noise and interference we define our receive signal as

$$
\underline{\mathrm{y}}=\underline{\mathrm{s}} * \underline{\mathrm{t}}+\underline{\mathrm{n}}+\underline{\mathrm{i}}
$$

where $\mathrm{t}$ is the extended target response (described in II-C), $\underline{\mathrm{n}} \in \mathbb{C} \overline{\mathbb{N}}\left(o, \sigma^{2}\right)$ is system noise, $\mathrm{i}$ is the interference described in the next section. We further process the received signal by projecting onto the transmit signal subspace, this gives

$$
\underline{\mathrm{s}}^{H} \underline{\mathrm{y}}=\underline{\mathrm{s}}^{H}(\underline{\mathrm{s}} * \underline{\mathrm{t}}+\underline{\mathrm{n}}+\underline{\mathrm{i}}) \text {. }
$$

The challenge then is to design $\underline{s}$ such that the impact to SINR performance is minimized by $\underline{t}$ and $\underline{i}$ while maintaining a constant modulus waveform.

\section{A. Waveform Design using ERA}

To design the waveform(s) (both the interrogate waveform to estimate the target response and novelly the TMI waveform), we will use the ERA. The ERA iteratively minimizes the error between the desired waveform (one with constant modulus and meeting the desired spectrum) and the designed waveform through the following steps. These steps are re-

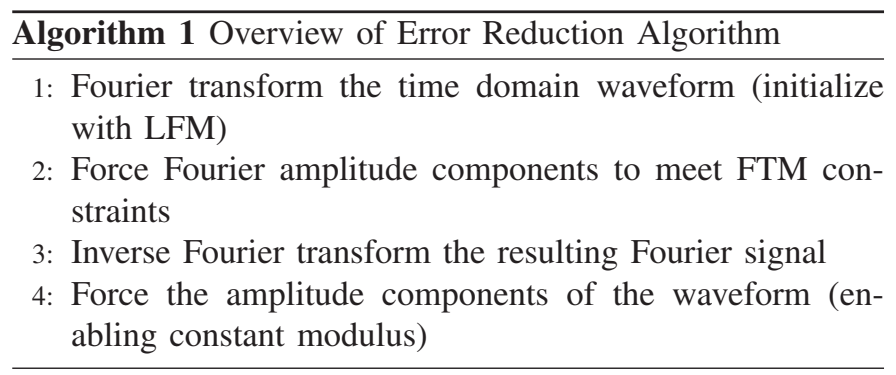

peated until a convergence criteria has been met (if possible), many good descriptions of this algorithm can be found in the literature [2], [3] and not worth detailing here.

Under the assumption that only constant modulus waveforms are permitted, the challenge in this paper is then to form the correct Fourier masks for the interrogate and TMI design stages in dynamic environments. The models chosen for the interference and target model are discussed next as well as the formation of these masks.

\section{B. Dynamic Interference Model}

In [4], [5] they develop a synthetic PSD model of RFI by employing a two-state Markov chain to define the spectral transitions from signal-and-noise present to noise-only and vice versa. They then modify the sequence with signal and noise specific amplitude distributions to arrive at the synthetic PSD.

For our purposes we will create two unique instantiations of the synthetic PSD. Each instantiation could represent a geographically varying environment or a change in the RFI over time. To create the time varying scenario we interpolate from the first instantiation to the second instantiation in the Fourier domain. The model can be described as follows;

$$
\underline{\mathrm{PSD}}= \begin{cases}\left(\underline{\mathrm{X}}_{\hat{\mathrm{P}}}+1\right) \odot \underline{\hat{f}}^{n}, & \text { if } \underline{\mathrm{X}}_{\hat{\mathrm{P}}}=0, \text { for } \forall m \\ \underline{\mathrm{X}}_{\hat{\mathrm{P}}} \odot \underline{\hat{f}}^{s}, & \text { for each signal band }\end{cases}
$$

where $\hat{f}^{n} \sim G_{\alpha^{n}, \beta^{n}, \gamma^{n}}(x), \hat{f}^{s} \sim G_{\Theta^{s}, \beta^{s}, \Lambda^{s}}(x)$ with $\Theta^{s} \sim$ $U\left[\alpha_{\min }, \alpha_{\max }\right], \Lambda^{s} \sim U\left[\log _{10}\left(\gamma_{\min }\right), \log _{10}\left(\gamma_{\max }\right)\right]$ and $\beta=$ $\frac{\Lambda^{s}}{\Theta^{s}}$. We point out that each signal band is an independent draw 


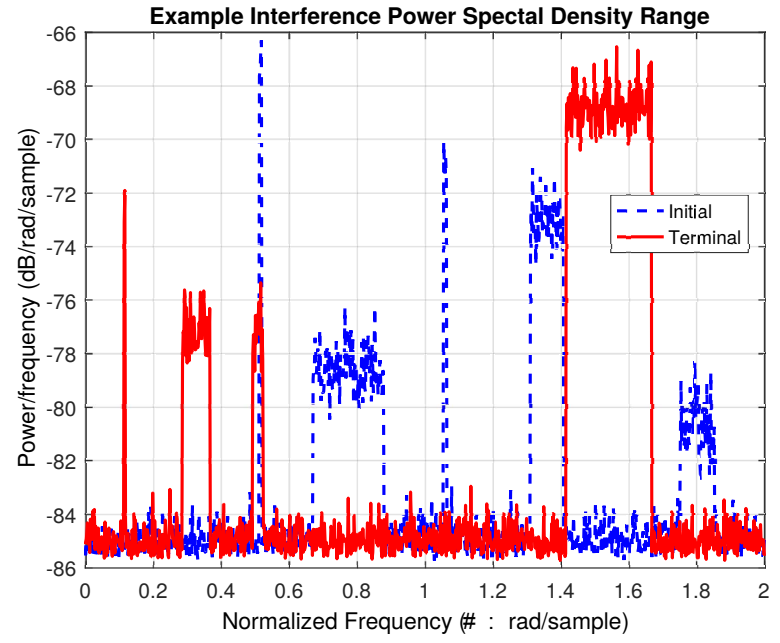

Fig. 2: Example RFI initial and terminal spectrum during a time period modelled by (4) with transition matrix (5).

of the random variable allowing for different mean-amplitude and variance.

The transition matrix for generating the synthetic RFI is

$$
\hat{\mathbf{P}}=\left[\begin{array}{ll}
0.9944 & 0.0056 \\
0.0296 & 0.9704
\end{array}\right] \text {. }
$$

An example RFI spectrum is shown in Figure 2 and we observe variation in the mean-amplitude for the signal bands (this could represent either power variation of the emitters in the scene or varied distances from the receiver) efficient usage of the signal band power.

Simplistically, though realistically any statistically stationary model could be used in its place, we think of the time varying (slowly varying) interference model as

$$
\underline{i}(m)=(M-m)\left(\underline{\mathbf{I}}_{1}(m)+\underline{n}_{1}(m)\right)+\left(\underline{n}_{2}(m)+m \underline{\mathbf{I}}_{2}(m)\right)
$$

where $\underline{m}=0,1,2, \cdots, M, M$ is total number of timesteps in the scenario, $\underline{I}_{1,2}$ is the interference environment whose PSD is represented by (4) and $\underline{n}_{1,2}$ is i.i.d. with distribution $\mathbb{C N}(0, \sigma)$.

This stage of the strategy will be considered executed after thresholding delineates between signal and noise. In our process we make use of the Minimum Description Length (MDL) [6] to estimate the rank of the signal subspace to create a binary FTM that allows us to describe the PU occupancy bands. The mask is, then, a set of amplitude values for each the frequency bins of the waveform. In this case, the bin is set to 0 if an interferer is present at that frequency band and 1 is only noise present. The mask is then used in the waveform design to dictate the waveform spectrum shape.

\section{Dynamic Target Model}

Previous works have used the ERA to design waveforms in the presence of coloured interference, [7], [8]. In our strategy, we perform inherent SINR optimization by target matched illumination while avoiding the interference. To accomplish, we first generate a notched LFM waveform with notches corresponding to the PU occupied bands. This intermediate designed signal can be generated using the ERA. The waveform would then be transmitted in order to estimate the target spectral response in the transmission bands (i.e. those not occupied by the PU).

The dynamic target is then modelled using a high modelorder auto-regressive process, described as;

$$
X(k)=\sum_{p=1}^{P} \phi(p) X(k-p)+\epsilon(k),
$$

where $P$ is the model order, $k$ the lag position, $\phi_{p}$ the model coefficients and $\epsilon_{k}$ the driving white noise with distribution $\mathbb{C N}(0, \sigma)$. This all-pole model (example in Figure 3) will generate spectrally peaky RF, representing extended targets with multiple scattering centres. Again, as described in the dynamic interference, we design an initial target response and a terminal response that varies over time through interpolation from one power spectrum to another power spectrum in each frequency sample. The received signal of the notched LFM

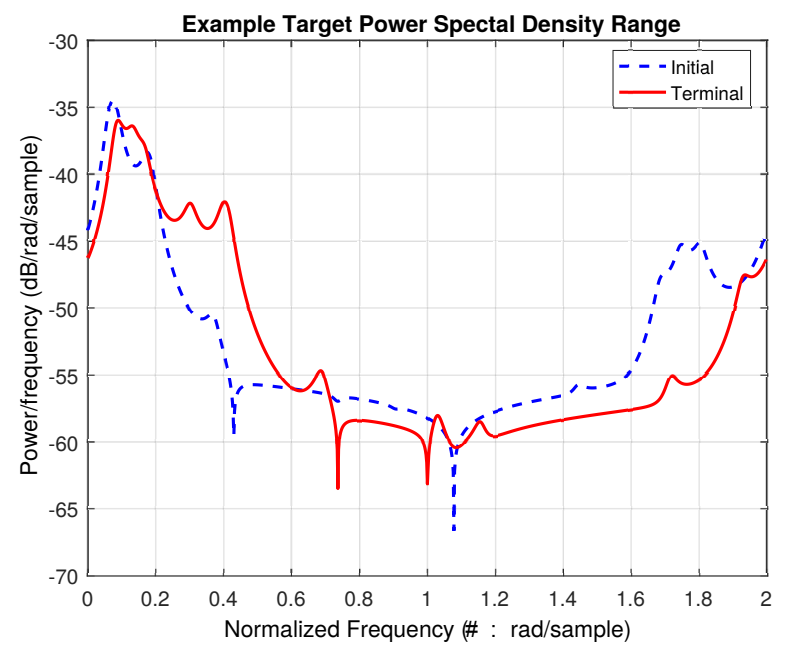

Fig. 3: Target Fourier response based on extended target assumption, modelled by AR process.

can be used to estimate the target spectral response where, in a similar manor as the interference, a Fourier mask is designed such that the peak target response is normalized to 1 with all other included frequency bins scaled accordingly. This will give a frequency dependent Fourier mask that will emphasize high power target returns and de-emphasize low power returns. Thus providing efficient use of transmit power across the spectrum.

Since the target response is dynamic (potentially changing aspect relative to the radar during the engagement) the mask will need to update periodically. For this experiment we will include a fixed update rate but, in future strategies the update rate should be adaptive based on the SINR loss.

After interference and target spectrum estimation, we desire to adapt the waveform to increase SINR for detection 
purposes. To accomplish, we form a joint FTM (simulated example in Figure 4) that includes the interference and target response.

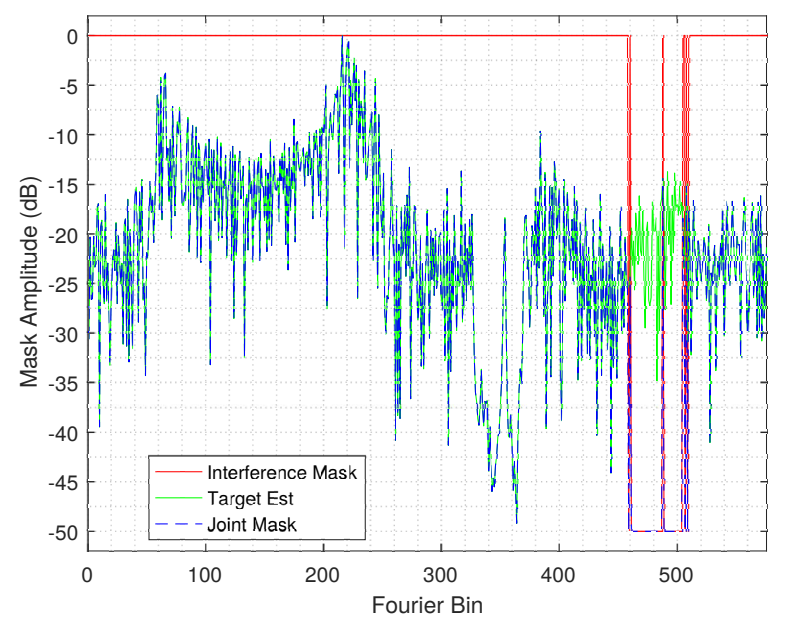

Fig. 4: Example FTM for the interference and joint interference and target masks.

\section{Simulation Results And Initial EXPERIMENTATION}

In this following sections we give the simulation results and initial experimental results. Both the simulation and experimental parameters are listed in Table I. Essentially,

TABLE I: Simulation and Experimental Parameters

\begin{tabular}{|c|c|}
\hline Parameter & Value \\
\hline Bandwidth (Hz) & $1.0 \mathrm{e} 9$ \\
\hline Carrier Freq (GHz) & 92.48 \\
\hline Coherent Pulses & 1024 \\
\hline Number CPI & 200 \\
\hline Waveform length (chips) & 576 \\
\hline PRF (Hz) & $10 \mathrm{e} 3$ \\
\hline Interference Update Rate (msec) & 512 \\
\hline Target Update Rate (msec) & 1024 \\
\hline
\end{tabular}

the simulation and data collects emulate a 20 second highly dynamic environment where the interference is re-estimated approximately twice a second and the target every second. These values have been chosen arbitrarily to display the concept but should, in practice, be more formally decided. The waveforms are updated and adapted to the environment and the waveforms and metrics such as SINR, spectral occupancy of the interference and the autocorrelation sequence of the waveforms are recorded for analysis. One note, the simulated interference and target response displayed are different to show unique cases.

\section{A. Simulation Results}

In Figure 7 we give simulated results displaying each step of our adaptive process. We observe the simulation displays the SINR consequence of the revisit rate for the target interrogation step, during which the notched LFM waveform is transmitted. We note the drop in SINR during this stage but also the significant improvement in the range sidelobes when compared to the SINR optimized TMI waveforms.

\section{B. Experimental Results}

The experimental realization of schemes, such as that described in this work, requires the availability of highly capable, highly flexible hardware and software systems, such as the CREW [9]. The system consists of 4 channels enabling arbitrary waveform generation, clock synchronization, a baseband converter responsible for up and down conversion to/from the intermediate frequency centred on $5.5 \mathrm{GHz}$, and the RF frequency of $92.48 \mathrm{GHz}$ with 4 channels of signal digitization, and the computer which controls the system. Each transmit and receive channel provides $1 \mathrm{GHz}$ of instantaneous bandwidth - the entirety of which is used in this experiment. The independent nature of the transmit and receive heads allow for spatially diverse experiments such as this.

The arrangement of transmit and receive heads for the experiments in this work is shown in Figure 8. One Tx/Rx pair of heads form the primary radar, a second pair implement the target simulator, and a single transmit head implements the environmental interference, which represents the primary user transmissions.

The data transfer load implied by the operational parameters mean the system will not run in real time, and will instead implement a stop-frame approach whereby after each radar transmission - target simulator reception cycle, and each target simulator transmission radar reception cycle, the system is frozen, data is transferred to the computer, processing is carried out and results returned to the hardware, and the system resumed.

We have included the results from two different set-ups of the experiment. The first is the more traditional non-target matched illumination approach but with applied notches for the estimated interference, this is represented in Figure 6. Second, we display the results, for the same interference and target instantiations, but including the TMI approach, shown in Figure 7. Note the significant SINR increase, but again, we note the cost being the degrade range sidelobes of the TMI waveform.

\section{CONCLUSION}

To conclude, despite very few research contributions marring the theoretical with simulated and experimental validation, we have proposed, and given simulation results and initial experimental results, an adaptive and autonomous signalling strategy to operate in a contested environment while considering modulus and target matching concerns for SINR. We have shown a novel use for the ERA in radar waveform design for TMI. We have observed the significant improvement in SINR over non-adaptive waveform strategies but at a cost of higher sidelobes. Additionally, the null depths of the designed 

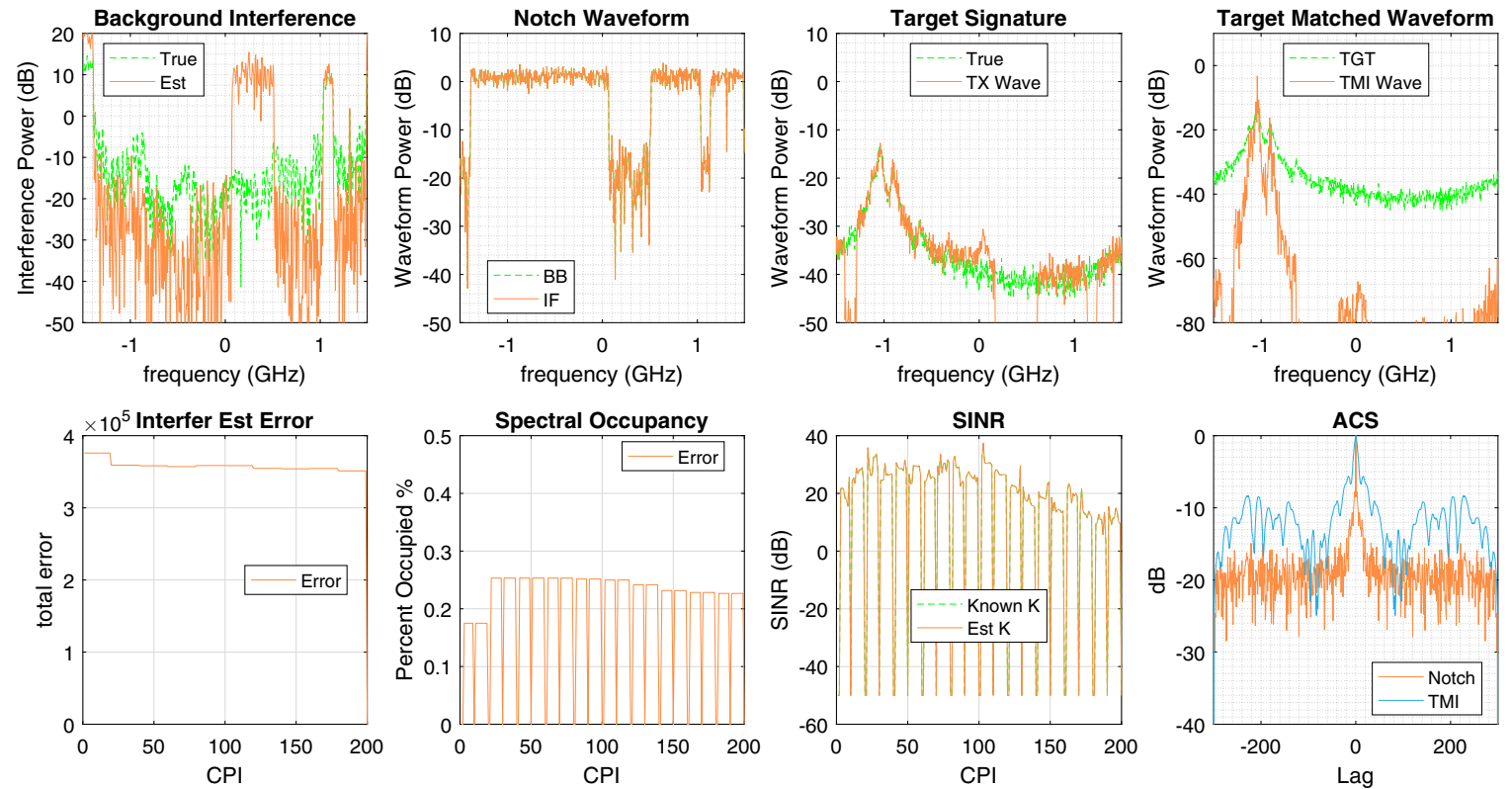

Fig. 5: Simulation capture for each stage (listen, interrogate, TMI) and evaluation metrics (interference estimation error, spectral occupancy, SINR and ACS). This simulation capture represents 200 CPI worth of simulated data for the dynamic interference and dynamic target model scenario.

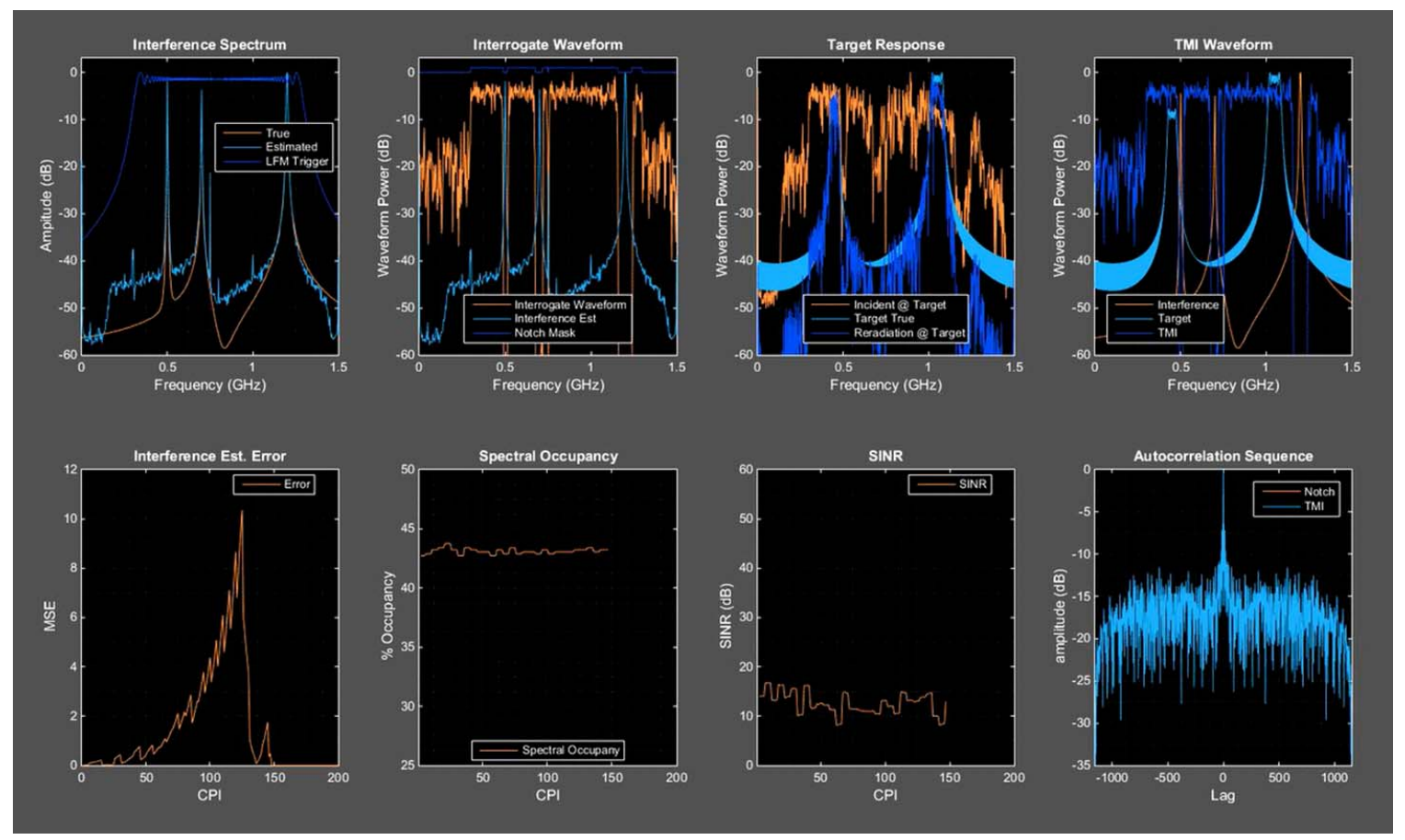

Fig. 6: Experimental results on CREW hardware of notch waveform only transmission. 


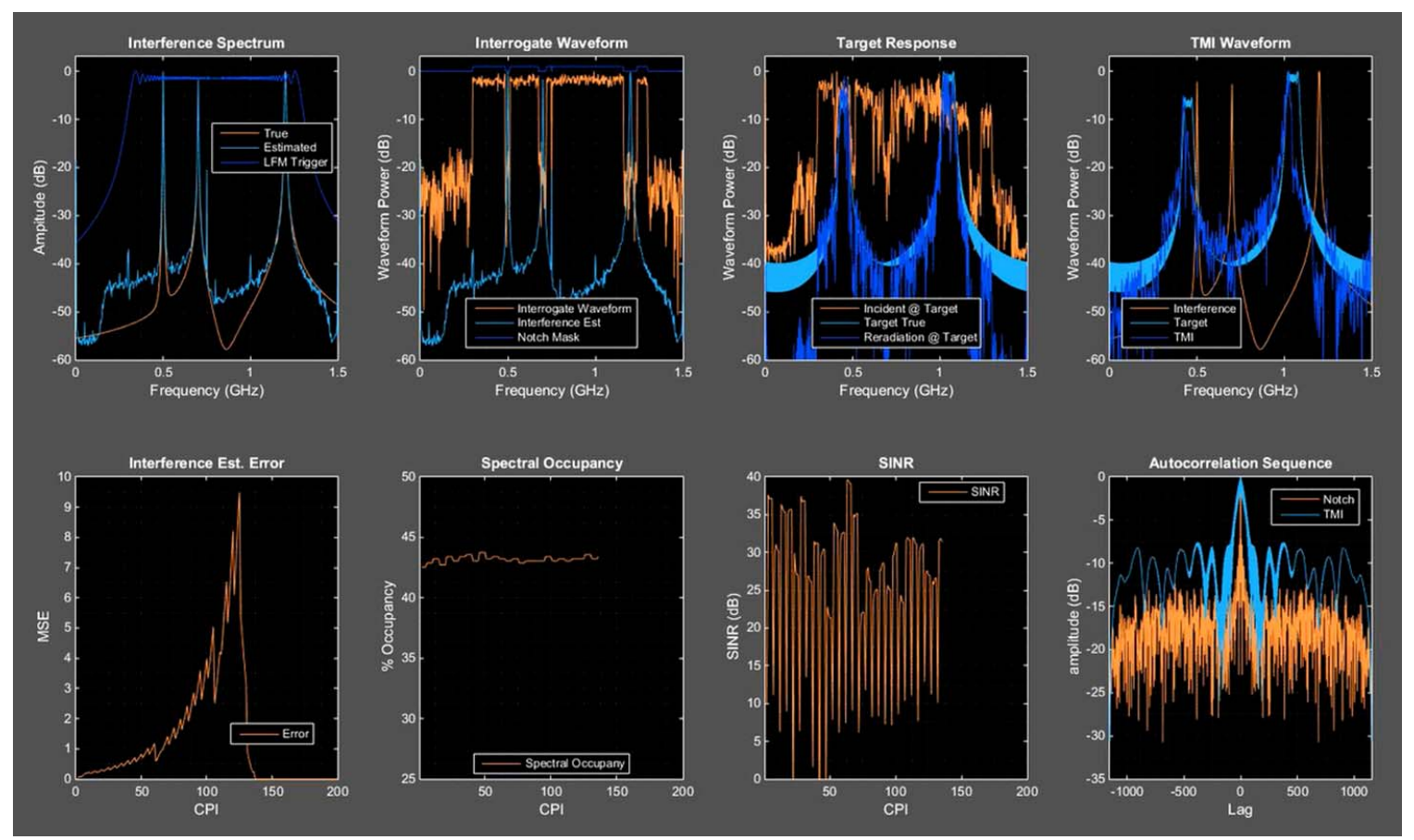

Fig. 7: Experimental results on CREW hardware of interference and TMI adaptive signal strategy.

\section{Radar Tx/Rx}

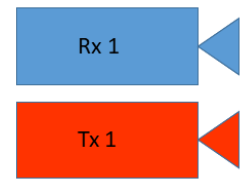

Target Simulator Tx/Rx
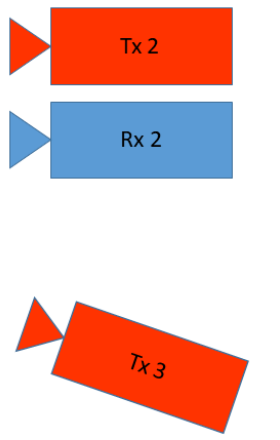

Environmental Interference Tx

waveforms using ERA are not suitable for simultaneous communications and radar suggesting future work to suppress the nulls depths and sidelobes.

\section{ACKNOWLEDGEMENT AND DISCLAIMER}

The authors would like to acknowledge both Mr. Richard Walkerdine and Mr. Jakob DeLong for their helpful discussions and collaborations regarding the development of the experimental trials.

Content includes material subject to Crown copyright (2018), Dstl. This material is licensed under the terms of the Open Government License except where otherwise stated. To view this license, visit http://www.nationalarchives.gov.uk/doc/open-government-

licence/version/3 or write to the Information Policy Team, The National Archives, Kew, London TW9 4DU, or email: psi@nationalarchives.gsi.gov.uk

\section{REFERENCES}

[1] R. Palama, H. Griffiths, and F. Watson, "A radar architecture for joint dynamic spectrum access and target-matched illumination," in IEEE Radar Conference. Seattle, Wash: IEEE, May 2017.

[2] J. Fienup, "Phase retrieval algorithms: a comparison," Appl. Opt., vol. 21, pp. 2758-2769, 1982.

[3] R. W. Gerchberg and W. Saxton, "A practical approach for the determination of the phase from image and diffraction plane pictures," Optik, vol. 35 , pp. 237-246, 1972.

[4] A. M. Jones, B. D. Rigling, and M. Rangaswamy, "Generation of synthetic uhf rfi in urban north american environments," in Proceedings of the 2016 IEEE Radar Conference. Philadelphia, PA: IEEE Xplore, 2016.

[5] A. M. Jones, "Performance prediction of constrained waveform design for adaptive radar," Ph.D. dissertation, Wright State University, June 2016.

[6] M. Wax and Kailath, "Detection of signals by information theoretic criteria," IEEE Transactions Acoust. Speech Signal Processing, vol. 33, no. 2, pp. 387-392, April 1985.

[7] L. Patton and B. Rigling, "Phase retrieval for radar waveform optimization," IEEE Transcations on Aerospace and Electronic Systems, vol. 48, no. 4, pp. 3287-3301, October 2012.

[8] L. Patton, S. Frost, and B. Rigling, "Efficient design of radar waveforms for optimised detection in coloured noise," IET Radar, Sonar and Navigation, vol. 6, no. 1, pp. 22-29, 2012.

[9] G. Smith, Z. Cammenga, A. Mitchell, K. L. Bell, M. Rangaswamy, J. T. Johnson, and C. Baker, "Experiments with cognitive radar," in IEEE 6th International Workshop on Computational Advances in Multi-Sensor Adaptive Processing (CAMSAP). IEEE, 2015, pp. 293-296. 